\title{
Verpasep Caltespen
}

National Cancer Institute

\section{Source}

National Cancer Institute. Verpasep Caltespen. NCI Thesaurus. Code C2811.

A recombinant chimeric protein composed of the heat shock protein 65 (Hsp65) from Mycobacterium bovis, and the human papilloma viral (HPV) protein E7. Hsp65, similar to other members of its family of proteins, elicits a strong immune response and may be used to design vaccines against a number of different cancers. E7 protein is involved in carcinogenesis of anal and cervical tumors, and represents a tumor antigen that may be specifically targeted by lymphocytes. ( $\mathrm{NCl04)}$ 